\title{
Forecasting the Internet of Things Market by Using the Grey Prediction Model Based Forecast Method
}

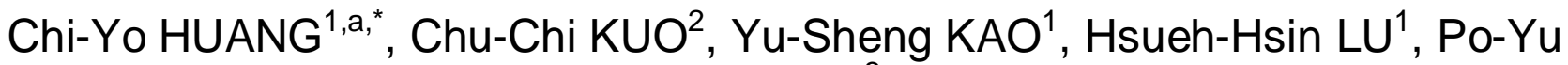 \\ $\mathrm{CHIANG}^{3}$
}

${ }^{1}$ Department of Industrial Education, National Taiwan Normal University, Taiwan

2 biLivingTek, INC, Hsinchu, Taiwan

${ }^{3}$ Institute of Communications Engineering, National Tsing Hua University, Taiwan

acyhuang66@ntnu.edu.tw

${ }^{*}$ Corresponding author

Keywords: Internet of Things (IoT), Technological Forecasting, Grey forecasting method, GM(1,1).

\begin{abstract}
The Internet of Things (IOT), extensions of the Internet into everything in the real world, enables computation all over the world. Albeit important, very few studies intended to predict the IoT market. In order to forecast the IoT market effectively with very few data, the $\operatorname{GM}(1,1)$ based Grey forecasting model was introduced. Thus, the authors developed a GM $(1,1)$ Grey system based pilot study for future short term wind power forecasting. The accurate predictions of the IoT market by using the $\operatorname{GM}(1,1)$ Grey forecasting method can serve as the basis for market analysis as well as strategic planning. An empirical study based on the real 2007-2011 worldwide IoT market data being provided by a leading market research institute has demonstrated the efficiency of the $\operatorname{GM}(1,1)$ based forecast mechanism. In the future, the $\operatorname{GM}(1,1)$ based forecast mechanism can further be applied to the forecast problems based on very limited time spans. Based on the forecast results, the IoT market will be over $\$ 700$ billion in year 2020 . The forecast error is $2.58 \%$. The empirical study results being obtained in this research indicate that the proposed $\operatorname{GM}(1,1)$ based approach is a promising alternative for predicting the very short time series.
\end{abstract}

\section{Introduction}

With the fast development of cloud computing, Internet applications have become more ubiquitous in our daily life. The Internet of Things (IOT) is based on the computing concept that depicts a future where each physical object would be connected to the Internet and have the ability to recognize themselves to other devices. The term is precisely described with radio-frequency identification (RFID) as the method of communication, although it also may contain other sensor technologies, wireless technologies or QR codes. In other words, IOT can be regarded as an Internet and mobile communication network which connect any items with the Internet to implement information exchange and communication, furthermore, to implement intelligent recognition, positioning, tracking, monitoring and management [1]. Currently, The IOT has been extensively applied in variety of domains, including logistics, health care, ambient intelligence (home, office, and factory), and individual as well as social environment [2].

Forecasting is the process of making statements about events whose actual outcomes have not yet been observed. A commonly example might be estimation for some variable of interest at some specified future date. There are basically types of forecasting models such as time series, 
causal/econometric forecasting, judgmental and artificial intelligence, etc.... Forecasting serves many predicting information and it can help people to organize planning in the future and market decision. Technology forecasting attempts to predict the future characteristics of useful technological machines, procedures or techniques. Primarily, a technological forecast deals with the characteristics of technology, such as levels of technical performance, like speed of a military aircraft, the power in watts of a particular future engine, the accuracy or precision of a measuring instrument, etc. The forecast does not have to state how these characteristics will be achieved. Secondly, technological forecasting usually deals with only useful machines, procedures or techniques. This is to exclude from the domain of technological forecasting those commodities, services or techniques intended for luxury or amusement. Commonly adopted methods of technology forecasting include the Delphi method, forecast by analogy, growth curves and trend extrapolation. Normative methods of technology forecasting such as the relevance trees, morphological models, and mission flow diagrams are also commonly used. Although there are manifold forecasting methods, several surveys have reported that trend extrapolation and Delphi, a structured group communication process for developing consensus from an expert group, are the most widely adopted for practical usage [3]. While the two methods have been commonly used inmost forecasting projects, they differ crucially in that trend extrapolation is a typical quantitative method, and on the contrary, Delphi is designed to solicit expert opinion qualitatively [4]

The Grey theory, a theory being developed originally by Deng [5] is a truly multidisciplinary and generic theory that deals with systems that are characterized by poor information and/or for which information is lacking [6]. Due to the nature of the short term wind speed prediction problem which is in lack of plenty information for forecasting, the authors developed a GM $(1,1)$ Grey system based pilot study for future short term wind power forecasting. The accurate predictions of wind speed by using the $\operatorname{GM}(1,1)$ Grey forecasting method can serve as the basis for introducing the wind power as an efficient source of alternative energies.

An empirical study based on the real worldwide IoT revenu will be provided for verifying the $\operatorname{GM}(1,1)$ based forecast mechanism. Based on the empirical study results, the wind speed can be precisely predicted based on the wind speed data of prior three hours.

This research is organized as follows. The related literature regarding to technological forecasting of market growth will be reviewed in Section 2. The GM $(1,1)$ Grey forecasting method will be introduced in Section 3. A prediction of the IoT market by the GM $(1,1)$ Grey forecasting method will be presented in Section 4. Discussions of the forecast results as well as future research possibilities will be presented in Section 5. Finally, the whole article will be concluded in Section 6.

\section{Technological Forecasting of Market Growth}

Technology forecasting is looking forward in an effort to anticipate the nature and significance of technology development before they occur. First, this paper reviews the step of the diffusion of innovations. Next, this paper introduces and surveys the common models and methods of technology forecasting. The predictive problems of time series belong to the area of technology forecasting, such as the stock prediction, power loading and semiconductor growth rate, etc. Final, there are some introductions of technological forecasting of diffusion of innovations in this section.

\section{Diffusion of Innovation}

Diffusion of Innovations is a theory that seeks to explain how, why, and at what rate new ideas and technology spread through cultures. Rogers [7] said diffusion is the process by which an innovation is communicated through certain channels over time among the members of a social system. The origins of the diffusion of innovations theory are varied and span across multiple disciplines. The process of diffusion begins with introduction of the innovation to the population, and ends when the population becomes saturated with adoptions. The primary tool for analysis of 
diffusion patterns is the cumulative adoption curve, which shows the percentage of a population that has adopted at any given point during the diffusion process [8].

There are some key elements in diffusion research which are: innovation, communication channels, time and social system. Innovation generally refers to the creation of better or more effective products, processes, technologies, or ideas that are accepted by markets, governments, and society. There are some characteristics of innovation will affect their rate of adoption. These characteristics include relative advantage, compatibility, complexity, trialability and observability. In the process of innovation, the use of communication between the individual and the group spread its spread can distinguish between homogeneity and heterogeneity, the most important concept is that both sides view this innovative product can be different (heterogeneous), but the level of education and degree preferably the same (homogeneous), this spread is the best. The innovation-decision period is the length of time required to pass through the innovation-decision process [8]. Social system can distinguish between the formal structure and informal structure, and the specification of system also cause obstacle to diffusion innovation.

Rogers [8] defines the five stages: knowledge, persuasion, decision, implementation, and confirmation. In knowledge stage, the individual is first exposed to an innovation but lacks information about the innovation. During this stage of the process the individual has not been inspired to find more information about the innovation. In persuasion stage, the individual is interested in the innovation and actively seeks information about the innovation. In decision stage, the individual takes the concept of the innovation and weighs the advantages/disadvantages of using the innovation and decides whether to adopt or reject the innovation. Due to the individualistic nature of this stage Rogers notes that it is the most difficult stage to acquire empirical evidence [8]. In implementation stage, the individual employs the innovation to a varying degree depending on the situation. During this stage the individual determines the usefulness of the innovation and may search for further information about it. In confirmation stage, the individual finalizes their decision to continue using the innovation and may use the innovation to its fullest potential.

There are two factors which determine the type of a particular decision. One concept is whether the decision is made freely and implemented voluntarily, and another is who makes the decision. Based on these considerations, three types of innovation decisions have been identified within diffusion of innovations which are optional innovation decision, collective innovation decision, and authority innovation decision. Optional innovation decision is made by an individual who is in some way distinguished from others in a social system. Collective innovation decision is made collectively by all individuals of a social system. Authority innovation decision is made for the entire social system by few individuals in positions of influence or power.

\section{Forecasting}

Forecasting is the process of making statements about events whose actual outcomes have not yet been observed. A commonly example might be estimation for some variable of interest at some specified future date. There are basically types of forecasting models such as time series, causal/econometric forecasting, judgmental and artificial intelligence, etc.

Time series method is an ordered sequence of values of a variable at equally spaced time intervals. Predicting is continuous of historical pattern such as the growth in sale, gross national product or stock market analysis. The example of time series methods are moving average, weighted moving average, exponential smoothing, autoregressive moving average (ARMA), autoregressive integrated moving average (ARIMA), extrapolation, linear prediction, trend estimation and growth curve, etc. Time Series Analysis is used for many applications such as: economic forecasting, sales forecasting, budgetary analysis, stock market analysis, inventory studies, workload projections and so on.

Causal/econometric forecasting methods forecasting methods use the assumption that it is possible to identify the underlying factors that might influence the variable that is being forecast. 
For example, including information about weather conditions might improve the ability of a model to predict umbrella sales. The examples of causal forecasting methods are regression analysis and autoregressive moving average with exogenous inputs (ARMAX), etc. Regression analysis includes a large group of methods that can be used to predict future values of variable using information about other variables. Linear regression applications are adopted almost every fields, engineering, physical sciences, economics management and so on [9]. Wiener [10] interested the ideas of functional series representation and further developed an existingmaterial in non-linear regression. Judgmental forecasting methods incorporate intuitive judgements, opinions and subjective probability estimates. This method does not require data in the same manner as quantitative forecasting methods and are mainly the product of judgment and accumulated knowledge. Qualitative techniques can be used in a wide range of circumstances. In some cases quantitative techniques cannot be used, e.g. when the past information about the values being forecast does not exist. For example, for a new product, there are no past data on sales on which to base estimates of future sales. The examples of Judgmental forecasting methods are composite forecasts, surveys, Delphi method, scenario building, technology forecasting and forecast by analogy, etc. The well known artificial intelligence methods are artificial neural networks (ANN) and Support vector machines (SVM).

To ensure the forecasting accuracy, every forecasting method should be measured with an appropriate measuring error function. The commonly used error functions are Mean Absolute Error (MAE), Mean Absolute Percentage Error (MAPE), Mean squared error (MSE), and Root Mean squared error (RMSE), etc.

\section{Technology Forecasting}

Forecasting serves many predicting information and it can help people to organize planning in the future and market decision. Technology forecasting attempts to predict the future characteristics of useful technological machines, procedures or techniques. Primarily, a technological forecast deals with the characteristics of technology, such as levels of technical performance, like speed of a military aircraft, the power in watts of a particular future engine, the accuracy or precision of a measuring instrument, etc. The forecast does not have to state how these characteristics will be achieved. Secondly, technological forecasting usually deals with only useful machines, procedures or techniques. This is to exclude from the domain of technological forecasting those commodities, services or techniques intended for luxury or amusement. Commonly adopted methods of technology forecasting include the Delphi method, forecast by analogy, growth curves and trend extrapolation. Normative methods of technology forecasting such as the relevance trees, morphological models, and mission flow diagrams are also commonly used. Although there are manifold forecasting methods, several surveys have reported that trend extrapolation and Delphi, a structured group communication process for developing consensus from an expert group, are the most widely adopted for practical usage [3]. While the two methods have been commonly used inmost forecasting projects, they differ crucially in that trend extrapolation is a typical quantitative method, and on the contrary, Delphi is designed to solicit expert opinion qualitatively [4].

The two main philosophical approaches to technology forecasting are exploratory forecasting and normative forecasting [11], [12]. The exploratory approach projects the present state of technology forward from the past/present situation. By contrast, the normative approach trace backward from a hypothetical future situation to assess its likelihood, timing and consequences. Some forecasting methods can be both exploratory and normative; methods would be better classified as to how useful they are in practical real-world prediction [12]. The commonly used exploratory forecasting methods are trend extrapolation, environment scanning, bibliometric analysis, and historical analogy. The typical normative forecasting methods, roadmapping and morphology analysis have been actively adopted in both practical and academic use. Many technology forecasting methods, 
including popular approaches such as Delphi, theory of inventive problem solving (TRIZ) and scenario, have been used for both exploratory and normative forecasting [12].

\section{Analytic Framework for the GM(1,1) Grey Forecasting Method}

Since Deng [28] proposed the Grey theory, related models have been developed and applied to the real world problems. Similar to fuzzy set theory, The Grey theory based on the assumption that a system is uncertain, and that the information regarding the system is insufficient to build a relational analysis or to construct a model to characterize the system [29] is a feasible mathematical means that can be used to deal with systems analysis characterized by inadequate information. Fields covered by the Grey theory include systems analysis, data processing, modeling, prediction, decision-making, and control engineering [29]. Following, the definitions will be modified by the work of Tseng et al. [29] as a basis for the Grey forecasting of the short term wind speed prediction in this research.

The accumulated generation, inverse accumulated generation, and the grey modeling are three basic operations of the Grey forecast model (GM). The operations of accumulated generation are used in the Grey forecast model to build differential equations. Intrinsically speaking, the Grey forecast model demonstrates the characteristics of requiring less data.

The $\operatorname{GM}(1,1)$ grey model, i.e., a single variable first-order grey model, is summarized as follows [30]:

Step 1: the initial sequence is $x^{(0)}=\left(x^{(0)}(1), x^{(0)}(2), \cdots, x^{(0)}(i), \cdots, x^{(0)}(n)\right)$, where $x^{(0)}(i)$ is the time series data at time $i$.

Step 2: based on the initial sequence $x^{(0)}$, a new sequence $x^{(1)}$ is generated by the accumulated generating operation (AGO), where $x^{(1)}=\left(x^{(1)}(1), x^{(1)}(2), \cdots, x^{(1)}(i), \cdots, x^{(1)}(n)\right)$ and $x^{(1)}(k)$ can be derived as follows: $x^{(1)}(k)=\sum_{i=1}^{k} x^{(0)}(i)$.

Step 3: the following first-order differential equation holds true: $\frac{d x^{(0)}}{d t}+a x^{(1)}=u$.

Step 4: from Step 3, $\hat{x}^{(1)}(k+1)=\left(x^{(0)}(1)-\frac{u}{a}\right) e^{-a k}+\frac{u}{a}, \quad \hat{x}^{(0)}(k+1)=x^{(1)}(k+1)-\hat{x}^{(1)}(k)$, where

$$
\hat{a}=\left[\begin{array}{l}
a \\
u
\end{array}\right]=\left(\mathbf{B}^{T} \mathbf{B}\right)^{-1} \mathbf{B}^{T} y_{N}, \quad \mathbf{B}=\left[\begin{array}{cc}
-0.5\left(x^{(1)}(1)+x^{(1)}(2)\right) & 1 \\
-0.5\left(x^{(1)}(2)+x^{(1)}(3)\right) & 1 \\
\vdots & \vdots \\
-0.5\left(x^{(1)}(n-1)+x^{(1)}(n)\right) & 1
\end{array}\right], \quad y_{N}=\left(x^{(0)}(2), x^{(0)}(3), \cdots, x^{(0)}(n)\right)^{T} .
$$

$\hat{x}^{(1)}(k+1)$ is the predicted value of $\hat{x}^{(1)}(k+1)$ at time $(k+1)$.

The GM $(1,1)$ grey model can easily be extended to the $\mathrm{GM}(1, \mathrm{~N})$ grey model. Note that the second index in the GM $(1, \mathrm{~N})$ grey model stands for $\mathrm{N}$ variables $\left(x_{1}^{(0)}, x_{2}{ }^{(0)}, \cdots, x_{N}{ }^{(0)}\right)$, and the differential equation can be written as follows: $\frac{d x_{1}^{(0)}}{d t}+a x_{1}^{(1)}=\sum_{i=2}^{N} b_{i}-x_{i}^{(1)}$, where $a, b_{1}, b_{2}, \cdots, b_{N-1}$ are unknown parameters. According to Step 4 of the GM $(1,1)$ grey model, these parameters can be estimated as follows: $\hat{a}=\left(\hat{a}, \hat{b}_{1}, \hat{b}_{2}, \cdots, \hat{b}_{N-1}\right)=\left(\mathbf{B}^{T} \mathbf{B}\right)^{-1} \mathbf{B}^{T} y_{N}$

where

$$
\mathbf{B}=\left[\begin{array}{cccc}
-0.5\left(x_{1}^{(1)}(1)+x_{1}^{(1)}(2)\right) & x_{2}^{(1)}(2) & \cdots & x_{N}^{(1)}(2) \\
-0.5\left(x_{1}^{(1)}(2)+x_{1}^{(1)}(3)\right) & x_{2}^{(1)}(3) & \cdots & x_{N}^{(1)}(3) \\
\vdots & \vdots & & \vdots \\
-0.5\left(x_{1}^{(1)}(n-1)+x_{1}^{(1)}(n)\right) & x_{2}^{(1)}(n) & \cdots & x_{N}^{(1)}(n)
\end{array}\right], \quad y_{N}=\left(x^{(0)}(2), x^{(0)}(3), \cdots, x^{(0)}(n)\right)^{T}
$$


The forecasts of $x_{1}^{(1)}$ can be derived by

$$
\hat{x}_{1}^{(1)}(k+1)=\left(x_{1}^{(0)}(1)-\sum_{i=2}^{N} \frac{b_{i}-1}{a} x_{i}^{(1)}(k+1)\right) e^{-a k}+\sum_{i=2}^{N} \frac{b_{i}-1}{a} x_{i}^{(1)}(k+1) .
$$

Finally, according to the work by Tseng et al [29], the post error test being introduced by Deng [30] was introduced to evaluate the accuracy of the grey forecasting. The forecasting errors were defined as $q^{(0)}(k)=x^{(0)}(k)-\hat{x}^{(0)}(k), k=1,2, \cdots, n$. The mean and the standard deviation of the forecasting errors are $\bar{q}$ and $S_{2}$. The mean and the standard deviation of the original time series can be derived by

$$
\bar{m}=n^{-1} \sum_{k=1}^{n} x^{(0)}(k), S_{1}=\sqrt{\sum_{k=1}^{n}\left(x^{(0)}(k)-\bar{m}\right)^{2} / n-1} .
$$

The post error ratio $C$ can be derived by dividing $S_{2}$ by $S_{1}$, or $C=S_{2} / S_{1}$. The lower the $\mathrm{C}$ is, the better the model is. The change rate of the forecasting error can be indicated by the post error ratio. Probability of small error is defined as $p=$ prob. $\left\{\left|q^{(0)}(k)-\hat{q}\right| \leq 0.6745 S_{1}\right\}$, where $k=2,3, \ldots, n[30]$. $p$ is another indicator of forecasting accuracy. This shows the probability that the relative bias of the forecasting error is lower than 0.6745. p is commonly required to be larger than 0.95 .

\section{Empirical Study}

In the following Section, the $\operatorname{GM}(1,1)$ model will be introduced for predicting the IoT market. An empirical study based on the 2007-2011 data from the work by iiMedia Research [31] which was measured from the real worldwide market data will be used to verify the feasibility of the $\operatorname{GM}(1,1)$. The prediction results as well as errors versus the original data were demonstrated in Table 1 and Figure 1.

\section{Discussion and Conclusions}

The Internet of Things (IOT), extensions of the Internet into everything in the real world, enables computation all over the world. Albeit important, very few studies intended to predict the IoT market. In order to forecast the IoT market effectively with very few data, the $\operatorname{GM}(1,1)$ based Grey forecasting model was introduced. The empirical study results being obtained in this research indicate that the proposed $\operatorname{GM}(1,1)$ based approach is a promising alternative for predicting the very short time series. In the future, the Grey Forecasting based methods can be applied in other emerging applications with very limited data.

Acording to the forecast results being demonstrated in Table 1and Fig. 1, the short term market growth of IoT can be predicted precisely, with the average error around $2.58 \%$ based on the historical worldwide IoT market data only of the prior four years. Based on the prediction results, the worldwide IoT market will be over $\$ 700$ Billion in year 2020. This pilot study based on the very limited historical IoT market data is apparently easier and especially suitable for the situations when the available data is very limited. In the future, introductions of other factors into the $\operatorname{GM}(1, N)$ model are worth future studies. A long term evaluation of the IoT prediction mechanism will be very helpful for verifying the feasibility of the applications of the Grey forecast method into the real world. Finally, future comparisons of the results being derived by the Grey forecast method with the results being derived by other artificial intelligence based approaches will also be very helpful for demonstrating the feasibility and efficiency of the methods. 


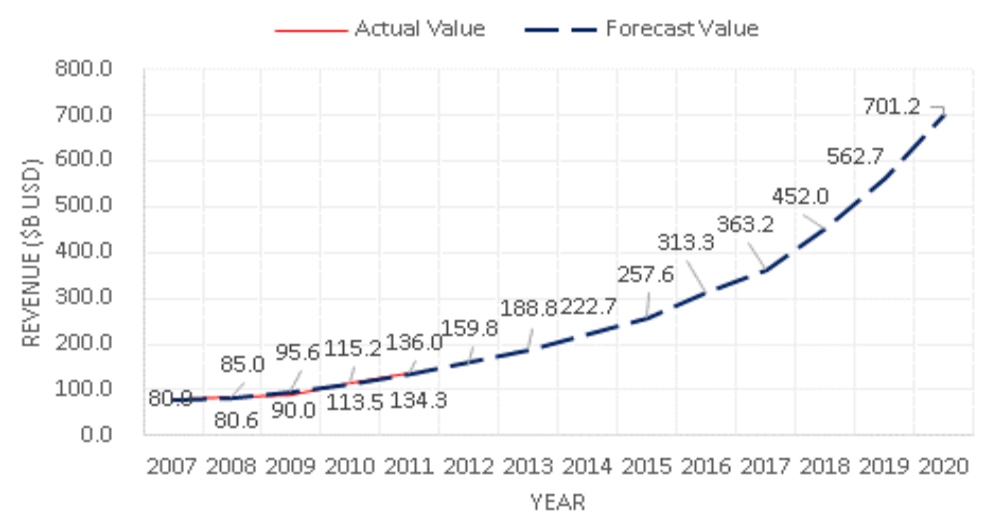

Figure 1. The Original versus the Prediction Results

Table 1.The Prediction Results and Errors Unit: \$B USD

\begin{tabular}{ccc}
\hline Year & Actual Value & Forecast Value \\
\hline 2007 & 80.0 & 80.0 \\
2008 & 85.0 & 81.1 \\
2009 & 90.0 & 96.0 \\
2010 & 115.2 & 113.5 \\
2011 & 136.0 & 134.3 \\
2012 & & 159.8 \\
2013 & & 188.8 \\
2014 & & 222.7 \\
2015 & & 257.6 \\
2016 & & 313.3 \\
2017 & & 363.2 \\
2018 & & 452.0 \\
2019 & & 562.7 \\
2020 & & 701.2 \\
\hline
\end{tabular}

\section{References}

[1] L. Atzori, A. Iera, G. Morabito, and M. Nitti, "The social internet of things (siot)-when social networks meet the internet of things: Concept, architecture and network characterization," Computer Networks, vol. 56, pp. 3594-3608, 2012.

[2] L. Atzori, A. Iera, and G. Morabito, "The internet of things: A survey," Computer networks, vol. 54, pp. 2787-2805, 2010. 
[3] J. P. Martino, "Technological forecasting—an overview," Management Science, vol. 26, pp. 28-33, 1980.

[4] B. Yoon and Y. Park, "Development of new technology forecasting algorithm: hybrid approach for morphology analysis and conjoint analysis of patent information," Engineering Management, IEEE Transactions on, vol. 54, pp. 588-599, 2007.

[5] J. Deng, "Grey system fundamental method," Huazhoug University of Science and Technology, Wuhan, China (in Chinese), 1985.

[6] C.-C. Hsu and C.-Y. Chen, "Applications of improved grey prediction model for power demand forecasting," Energy Conversion and Management, vol. 44, pp. 2241-2249, 2003.

[7] M. Rogers Everett, "Diffusion of innovations," New York, 1995.

[8] R. G. Fichman and C. F. Kemerer, "The illusory diffusion of innovation: An examination of assimilation gaps," Information Systems Research, vol. 10, pp. 255-275, 1999.

[9] F. Pesarin and J. Wiley, Multivariate permutation tests: with applications in biostatistics vol. 240: Wiley Chichester, 2001.

[10] N. Wiener, "Nonlinear problems in random theory," Nonlinear Problems in Random Theory, by Norbert Wiener, pp. 142. ISBN 0-262-73012-X. Cambridge, Massachusetts, USA: The MIT Press, August 1966.(Paper), vol. 1, 1966.

[11] M. Bengisu and R. Nekhili, "Forecasting emerging technologies with the aid of science and technology databases," Technological Forecasting and Social Change, vol. 73, pp. 835-844, 2006.

[12] B. Twiss, "Forecasting for technical decisions," Peter Peregrinus, London, 1992.

[13] F. M. Bass, "Comments on "a new product growth for model consumer durables the bass model"," Management science, vol. 50, pp. 1833-1840, 2004.

[14] A. S. Ehrenberg, M. D. Uncles, and G. J. Goodhardt, "Understanding brand performance measures: using Dirichlet benchmarks," Journal of Business Research, vol. 57, pp. 1307-1325, 2004.

[15] C. Skiadas, "Two generalized rational models for forecasting innovation diffusion," Technological Forecasting and Social Change, vol. 27, pp. 39-61, 1985.

[16] V. Mahajan and M. E. Schoeman, "Generalized model for the time pattern of the diffusion process," Engineering Management, IEEE Transactions on, pp. 12-18, 1977.

[17] A. W. Blackman Jr, "A mathematical model for trend forecasts," Technological Forecasting and Social Change, vol. 3, pp. 441-452, 1972.

[18] J. C. Fisher and R. H. Pry, "A simple substitution model of technological change," Technological forecasting and social change, vol. 3, pp. 75-88, 1972. 
[19] M. Nawaz Sharif and C. Kabir, "System dynamics modeling for forecasting multilevel technological substitution," Technological Forecasting and Social Change, vol. 9, pp. 89-112, 1976. [20] C. Easingwood, V. Mahajan, and E. Muller, "A nonsymmetric responding logistic model for forecasting technological substitution," Technological forecasting and Social change, vol. 20, pp. 199-213, 1981.

[21] M. N. Sharif and K. Ramanathan, "Binomial innovation diffusion models with dynamic potential adopter population," Technological Forecasting and Social Change, vol. 20, pp. 63-87, 1981.

[22] D. Nissim and S. H. Penman, "Ratio analysis and equity valuation: From research to practice," Review of accounting studies, vol. 6, pp. 109-154, 2001.

[23] S. H. Penman, "The articulation of price-earnings ratios and market-to-book ratios and the evaluation of growth," Journal of Accounting Research, pp. 235-259, 1996.

[24] E. F. Koenig and S. Dolmas, "Real-time GDP growth forecasts," 1997.

[25] E. F. Koenig, S. Dolmas, and J. Piger, "The use and abuse of real-time data in economic forecasting," Review of Economics and Statistics, vol. 85, pp. 618-628, 2003.

[26] J. P. LeSage, "Forecasting turning points in metropolitan employment growth rates using Bayesian techniques," Journal of Regional Science, vol. 30, pp. 533-548, 1990.

[27] J. P. LeSage and M. Magura, "Using Bayesian techniques for data pooling in regional payroll forecasting," Journal of Business \& Economic Statistics, vol. 8, pp. 127-135, 1990.

[28] D. Ju-Long, "Control problems of grey systems," Systems \& Control Letters, vol. 1, pp. 288-294, 1982.

[29] F.-M. Tseng, H.-C. Yu, and G.-H. Tzeng, "Applied hybrid grey model to forecast seasonal time series," Technological Forecasting and Social Change, vol. 67, pp. 291-302, 2001.

[30] J. Deng, "Grey forecasting and decision," Huazhong University of Science and Technology Press, Wuhan, pp. 97-134, 1986.

[31] V. Kecman, Learning and soft computing: support vector machines, neural networks, and fuzzy logic models: MIT press, 2001 